\title{
Research on the Thermo-mechanical Effect of Large-size Cables and its Influence on Insulation Based on Finite Element Method
}

\author{
Shengchong $\mathrm{Li}^{1}$, Fujue $\mathrm{Wang}^{1}$, Hongzhong $\mathrm{Ma}^{1}$, Honghua $\mathrm{Xu}^{2}$, and Chunning $\mathrm{Wang}^{2}$ \\ ${ }^{1}$ College of Energy and Electrical Engineering, Hohai University, Nanjing, 211100, China \\ ${ }^{2}$ State Grid Nanjing Power Supply Company, Nanjing, 210008, China
}

\begin{abstract}
In order to study the thermo-mechanical effect of large-size cables, the thermal-structural coupling model is established to calculate the stress and deformation of the cables based on finite element method. According to the model, the distribution characteristics of temperature, stress and deformation are analysed in this paper. It turns out that, the maximum stress appears in metal sheath and the largest axial deformation in insulation layer. By means of curve fitting, a quick formula is proposed to calculate the stress on metal sheath and the axial deformation of conductor. Finally, its influence on insulation is studied by the simulation of electric field. The results show that, the electric field strength on the surface of insulation layer increases significantly under this circumstance, leading to the occurrence of partial discharge.
\end{abstract}

\section{Introduction}

The thermo-mechanical effect generated during the operation of high-voltage and large-size cables is becoming a main reason for power cable faults [1-2]. It is mainly reflected in the stress and deformation of cables. While in steady-state operation, there is a power loss inside the cable. This part of loss will be converted into thermal energy, which leads to the temperature rise and thermal expansion of the cable. If stress and deformation cannot be released, it will cause damage to the structure of cable. Therefore, the research of thermo-mechanical effect and its influence helps to grasp the running status of cables, improve operational reliability and reduce fault ratio.

The research of cable temperature field is the basis of thermo-mechanical study. At present, there are mainly two approaches to calculate cable temperature, thermal circuit method and numerical calculation methods. Thermal circuit method is based on the IEC standard 60287 with a simple model and a fast calculation speed [3-4]. However this method is under some ideal assumptions, so the accuracy is not always satisfactory. Numerical methods include finite element method, boundary element method, and finite difference method [5-6]. Among them, the finite element method has been widely used because it is able to set mesh model and boundary conditions freely.

There are few studies on the distribution characteristics of stress and deformation of large-size cables in existing papers. In reference [7], the analytical method was used to calculate the axial thrust and thermal expansion of large-size cables. However, due to the simple model, the calculation accuracy is not satisfactory.
Reference [8] analysed the stress field distribution characteristics of the cleat for large-size cable by finite element method, but did not study the stress field inside the cable.

In this paper, the $220 \mathrm{kV}$ cable with a $2500 \mathrm{~mm}^{2}$ section is selected as the research object. Based on ANSYS, a thermal-structural coupling model is built for large-size cables. According to the results of calculation model, the distribution characteristics of temperature, stress as well as deformation are analysed in this paper. In addition, the electric field model of cable is established by Maxwell to analyse the influence of thermo-mechanical effect on insulating property.

\section{Analysis of temperature field}

\subsection{Temperature field model}

By reference to the parameters of ZC-YJLW03-Z-127 $220 \mathrm{kV}-1 \times 2500 \mathrm{~mm}^{2}$ cable, a simplified five-layer model is built in ANSYS, which consists of conductor, insulation layer, buffer layer (equivalent to insulation shielding and water barrier), metal sheath, and outer sheath. The parameters of structure and thermal property are listed in Table 1.

Table 1. The parameters of structure and thermal property

\begin{tabular}{|c|c|c|}
\hline Structure & $\begin{array}{c}\text { Out diameter } \\
/ \mathrm{mm}\end{array}$ & $\begin{array}{c}\text { Thermal conductivity } \\
/ \mathrm{W} \cdot \mathrm{m}^{-1} \cdot \mathrm{K}^{-1}\end{array}$ \\
\hline Conductor & 60.4 & 377 \\
\hline Insulation layer & 115.4 & 0.2857 \\
\hline Buffer layer & 127.4 & 0.23 \\
\hline Metal sheath & 133 & 230 \\
\hline
\end{tabular}




\begin{tabular}{|l|l|l} 
Outer sheath & 143 & 0.1667 \\
\hline
\end{tabular}

The temperature field model is based on the assumptions that each material is a continuous and homogeneous media and its thermal conductivity is isotropic. Tetrahedron is utilized as the mesh element in this model.

\subsection{Boundary conditions and load calculation}

According to the theory of heat transfer, end faces of the cable are under Neumann boundary condition with zero normal heat flux, and the external surface of outer sheath is under Robin boundary condition. In this model, the air temperature is $25^{\circ} \mathrm{C}$, and the convection film coefficient of air is $5.6 \mathrm{~W} /\left(\mathrm{m}^{2} \cdot \mathrm{K}\right)$.

During the operation of large-size cables, insulation layer loss is very small compared with conductor loss and metal sheath loss. Therefore, the model mainly considers conductor loss and metal sheath loss. These two parts of losses are applied to conductor and metal sheath as the internal heat generation, which can be calculated by the IEC standard 60287.

\subsection{Results of temperature field simulation}

The maximum operating temperature of the XLPE cable conductor cannot exceed $90^{\circ} \mathrm{C}$. Therefore, the load current applied in the simulation needs to meet the precondition. When the model is applied a $2200 \mathrm{~A}$ load current, the temperature field distribution result is shown in Figure 1. In this situation, conductor temperature is $89.74^{\circ} \mathrm{C}$, reaching the warning state, and outer sheath temperature is $54.25^{\circ} \mathrm{C}$, which is the lowest inside the cable.

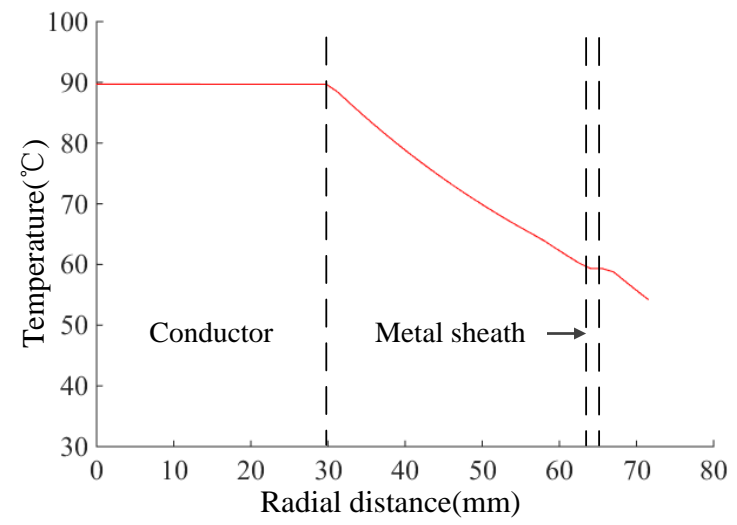

Fig. 1. The radial distribution curve of temperature under a 2200 A load current

It can be seen that the temperature inside the conductor and metal sheath are basically the same while a negative temperature gradient exists in each of the other layers. This is mainly because conductor and metal sheath have a large thermal conductivity.

\section{Analysis of thermo-mechanical effect}

\subsection{Basic theory of thermal-structural coupling}

The strain of each element is composed of thermal strain and the elastic strain caused by stress. For a node inside the cable, an equation can be built as follows:

$$
\mathrm{d} \boldsymbol{\sigma}_{\boldsymbol{t h}}=\boldsymbol{K} \mathrm{d} \boldsymbol{\delta}
$$

Where, $\mathrm{d} \boldsymbol{\sigma}_{\boldsymbol{t} \boldsymbol{h}}$ represents the temperature load increment of node, calculated by equation (2); $\boldsymbol{K}$ represents the stiffness matrix of node, calculated by equation (3); $\mathrm{d} \boldsymbol{\delta}$ represents the deformation increment of node.

$$
\begin{gathered}
\mathrm{d} \boldsymbol{\sigma}_{\boldsymbol{t h}}=\int \boldsymbol{B}^{T} \boldsymbol{C} \mathrm{d} T \mathrm{~d} V \\
\boldsymbol{K}=\int \boldsymbol{B}^{T} \boldsymbol{D} \boldsymbol{B} \mathrm{d} V
\end{gathered}
$$

Where, $\boldsymbol{B}$ represents the transfer matrix for strain $\boldsymbol{\varepsilon}$ and deformation $\boldsymbol{\delta}$, shown in equation (4); $\boldsymbol{C}$ represents a matrix associated with temperature, calculated by equation (5).

$$
\begin{gathered}
\mathrm{d} \boldsymbol{\varepsilon}=\boldsymbol{B} \mathrm{d} \boldsymbol{\delta} \\
\boldsymbol{C}=\boldsymbol{D}\left(\boldsymbol{\alpha}+\frac{\partial \boldsymbol{D}^{-1}}{\partial T} \boldsymbol{\sigma}_{t h}\right)
\end{gathered}
$$

Where, $\boldsymbol{\alpha}$ represents thermal expansion coefficient; $\boldsymbol{D}$ represents the elastic matrix, determined by elastic modulus and Poisson ratio.

\subsection{The thermal-structural model}

The cable structure model is still established as the five-layer model. Since the cable length between adjacent hoops is generally $3 \sim 5 \mathrm{~m}$, the model length is set as $3 \mathrm{~m}$ considering the calculation speed. The mechanical parameters of each layer of are listed in Table 2.

The simulation is performed under continuity hypothesis, homogeneity hypothesis, isotropy hypothesis and linear hypothesis. Fixed constraint conditions are not applied to the model.

Table 2. The mechanical parameters of each layer

\begin{tabular}{|c|c|c|c|}
\hline Structure & $\begin{array}{c}\text { Elastic } \\
\text { modulus } \\
/ \mathrm{MPa}\end{array}$ & $\begin{array}{c}\text { Poisson } \\
\text { ratio }\end{array}$ & $\begin{array}{c}\text { Thermal expansion } \\
\text { coefficient } \\
/ 10^{-5} \cdot \mathrm{K}^{-1}\end{array}$ \\
\hline Conductor & 121000 & 0.33 & 1.8 \\
\hline Insulation layer & 150 & 0.37 & 15 \\
\hline Buffer layer & 41.84 & 0.48 & 28 \\
\hline Metal sheath & 7000 & 0.33 & 2.4 \\
\hline Outer sheath & 200 & 0.39 & 26 \\
\hline
\end{tabular}

\subsection{Results of the thermal-structural coupling simulation}

The result of temperature field simulation under a 2200 A load current is coupled as the temperature load into the simulation of cable stress field. The magnitude of stress is characterized by Von-Mises stress, and the stress field of a cable section is shown in Figure 2. The maximum stress appears in metal sheath with a value of $96.39 \mathrm{MPa}$, while the stress in any other layer is far less than that in metal sheath. 


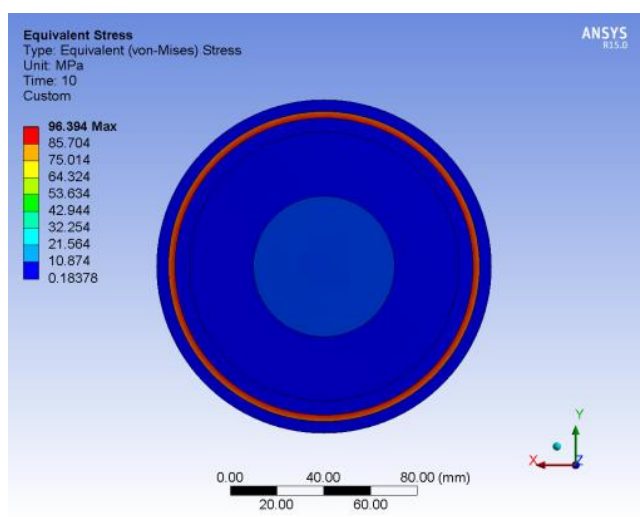

Fig. 2. The distribution graph of stress under a $2200 \mathrm{~A}$ load current

Considering the difference among the materials in expansion coefficient, elastic modulus, and temperature, the thermal deformation of insulation layer, buffer layer and outer sheath is relatively large. So the metal sheath is double-extruded to bear the maximum stress. According to the data, the running state of metal sheath needs to be paid more attention to.

Cable deformation mainly includes radial expansion and axial elongation. The axial deformation of cable under a 2200 A load current is shown in Figure 3.

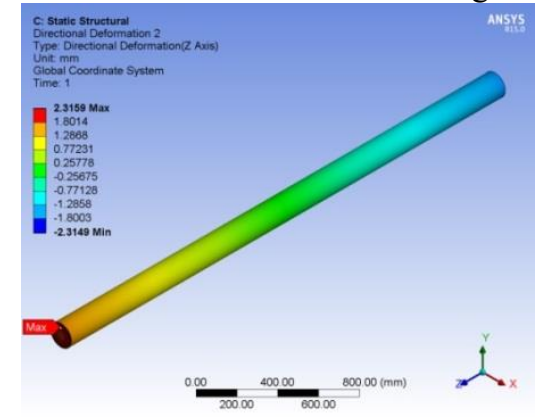

Fig. 3. The distribution graph of axial deformation under a 2200 A load current

It can be seen that the axial deformation cannot be neglected in this situation. Using the probe in the end face, the axial deformation of insulation layer is up to $2.316 \mathrm{~mm}$, and that of conductor and metal sheath is $1.844 \mathrm{~mm}$ and $1.602 \mathrm{~mm}$. The axial deformation can easily lead to the mechanical faults in the intermediate joints.

Out of the phenomenon of metal fatigue, deformation of metal material under an alternating stress is not easy to recover. Therefore, it is important to notice the conditions of conductor and metal sheath during operation. Figure 4 shows the distribution curve of radial deformation in a cable section.

From figure 4, the radial expansion of the whole body reaches $0.24 \mathrm{~mm}$ while that of insulation layer is nearly 0 . It indicates that insulation layer is under severe extrusion. As a result, when the cable runs a long time, the insulation layer tends to be damaged, probably causing an insulation fault.

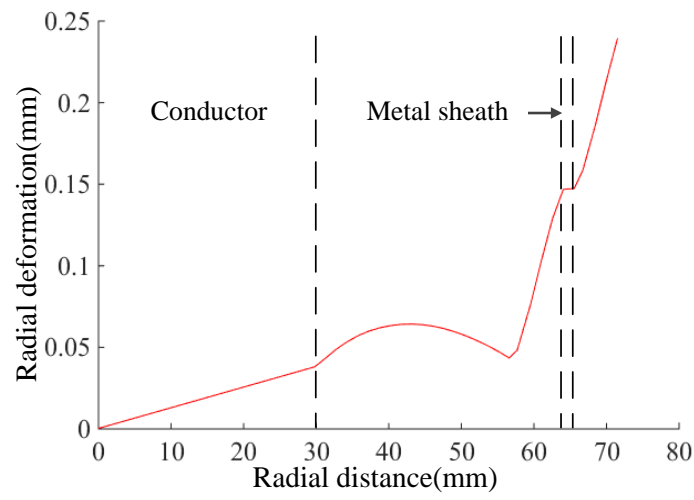

Fig. 4. The radial distribution curve of radial deformation under a 2200 A load current

\subsection{A quick calculation formula for stress and deformation}

There is an urge to provide the department of cable operation and maintenance with a quick calculation formula for stress and deformation under different load currents. The stress in metal sheath and the axial deformation of conductor are the most critical data, so the formula aims at these two parameters.

Thermo-mechanical effect is considered not negligible under a large current load, so the range of current loads varies from $800 \mathrm{~A}$ to $2200 \mathrm{~A}(\Delta I=100 \mathrm{~A})$. The corresponding data calculated by finite element method are listed in Table 3.

Table 3. The data under different current loads

\begin{tabular}{|c|c|c|}
\hline Load current/A & $\begin{array}{c}\text { Stress in metal } \\
\text { sheath/MPa }\end{array}$ & $\begin{array}{c}\text { Axial deformation of } \\
\text { conductor/mm }\end{array}$ \\
\hline 800 & 17.80 & 0.3216 \\
\hline 900 & 20.93 & 0.3832 \\
\hline 1000 & 24.44 & 0.4521 \\
\hline 1100 & 28.31 & 0.5282 \\
\hline 1200 & 32.56 & 0.6116 \\
\hline 1300 & 37.17 & 0.7022 \\
\hline 1400 & 42.16 & 0.8002 \\
\hline 1500 & 47.51 & 0.9050 \\
\hline 1600 & 53.23 & 1.018 \\
\hline 1700 & 59.32 & 1.137 \\
\hline 1800 & 65.78 & 1.264 \\
\hline 1900 & 72.60 & 1.398 \\
\hline 2000 & 79.81 & 1.540 \\
\hline 2100 & 87.40 & 1.688 \\
\hline 2200 & 95.36 & 1.844 \\
\hline
\end{tabular}

The polynomial fitting method is utilized to perform curve fitting. According to the fitting results, these two parameters are calculated by equation $(6) \sim(8)$.

$$
\begin{gathered}
\sigma_{\mathrm{V}}=3.7026 \bar{I}^{2}+24.768 \bar{I}+47.503 \\
x=0.072525 \bar{I}^{2}+0.48639 \bar{I}+0.90522 \\
\bar{I}=(I-1500) / 447.21
\end{gathered}
$$

Where, $\sigma_{\mathrm{V}}$ represents the stress in metal sheath; $x$ represents the axial deformation of conductor; $I$ represents the load current. 


\section{The influence on insulation}

\subsection{The electric field model}

In the simulation of electric field, it is necessary to define the conductivity and relative permittivity of material accurately. The relative permittivity of copper is 1 , and the conductivity is $5.8 \times 10^{7} \mathrm{~s} / \mathrm{m}$; the relative permittivity of XLPE is 2.5 , and the conductivity $\sigma_{\mathrm{XLPE}}$ can be calculated by an empirical formula which is widely acknowledged [9].

$$
\sigma_{\mathrm{XLPE}}=\sigma_{0} \exp \left(a \theta_{\mathrm{XLPE}}+b E\right)
$$

Where, $\sigma_{0}$ represents the conductivity in the situation where both temperature and electric field strength are 0 ; $a$ represents the temperature coefficient; $\theta_{\mathrm{XLPE}}$ represents the temperature of insulation layer; $b$ represents the electric field strength coefficient.

With the existence of insulation shielding, the model does not consider the electric field outside the insulation layer. This model is applied with default boundary conditions and a $311 \mathrm{kV}$ voltage as the excitation.

\subsection{Results of the electric field simulation}

After a long-term operation of cables under heavy loads, impressions often appear in the surface of insulation layer because of thermo-mechanical effect. An air gap is set in insulation layer to simulate the damage caused by thermo-mechanical effect. According to the cases of insulation fault, the air gap is set as an ellipse. Its long axis length is $10 \mathrm{~mm}$ and minor axis length is $2 \mathrm{~mm}$.

Electric potential and electric field strength are the most critical parameters in the electric field simulation. Figure 5 and Figure 6 show the radial distribution of electric potential and electric field strength in normal condition and damaged condition.

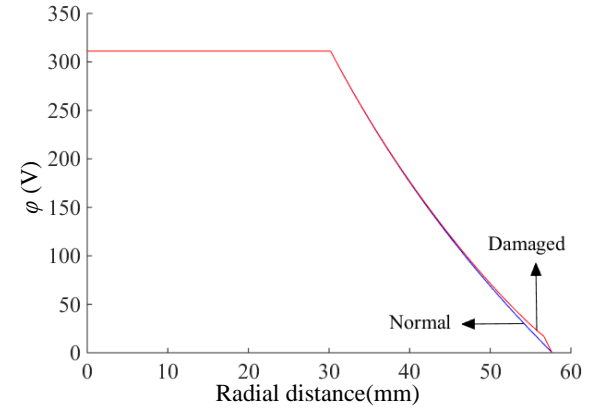

Fig. 5. The radial distribution curve of electric potential

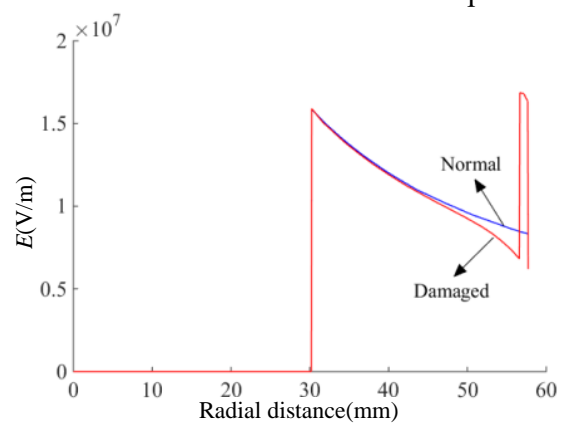

Fig. 6. The radial distribution curve of electric field strength
It can be seen that the air gap makes the electric potential in insulation layer increase, but the influence is not obvious. However, the influence of air gap on electric field is significant.

The electric field strength in the air gap increases sharply to $1.69 \times 10^{7} \mathrm{~V} / \mathrm{m}$, which exceeds the breakdown field intensity of air. That means a phenomenon of partial discharge occurs. If it is not well disposed of, there will be an insulation fault eventually. It comes to a conclusion that, thermo-mechanical effect will severely jeopardize the safe and stable operation of large-size cables.

\section{Conclusions}

Based on the analysis of this paper, conclusions are as follows:

1) The temperature inside the conductor and metal sheath are basically the same while a negative temperature gradient exists in each of the other layers; the maximum stress appears in metal sheath; the distribution of deformation is uneven, and the maximum axial deformation is in insulation layer.

2) The stress of metal sheath and axial deformation of conductor can be quickly calculated by a quadratic polynomial related to the load current.

3) The long-term thermo-mechanical effect brings damage to insulation layer. It will cause a sharp increase of electric field strength in certain locations, leading to a reduction of insulation property of cables.

This work is supported by Jiangsu Electric Power Company Science and Technology Project (J2017070) and "111" Project of "Renewable Energy and Smart Grid" (B14022).

\section{References}

1. Y.F. Dai, J.Y. Lu, X. Zhang, J. Wang, B.F. Zhu, IEEE Trans. Plasma Sci. 45, 1184 (2017)

2. Y.P. Liu, H.C. Liu, L.C. Yu, Y.D. Li, L.J. Gao, IEEE Trans. Dielectr. Electr. Insul. 24, 1355 (2017)

3. J.B. Duan, C.Q. Yin, A.Q. Lyu, Y.Q. Li, High Volt. App. 50, 1 (2014)

4. Y.C. Liang, High Volt. Eng. 42, 1142 (2016)

5. A. Sedaghat, F. De León, IEEE Trans. Power Del. 29, $2306(2014)$

6. X. Lu, A.X. Zhao, J.B. Deng, G.J. Zhang, P. Yu, S.J. Yao, CICED 2014, 1280(2014)

7. J.H. Luo, L. Zhang, Y.G. Liu, C.B. Zheng, High Volt Eng. 36, 1281 (2010)

8. H.Y. Liu, N. Liu, L.J. Pu, S.L. You, H. Wu, J. Li, Trans. China Electrotech. Soc. 31, 170 (2016)

9. Y.P. Hao, Y. Chen, L. Yang, W.H. Qiu, M.L. Fu, S. Hou, High Volt. Eng. 43, 3534 (2017) 